The Astrophysical Journal SuPPlement SER IEs, 90:547-552, 1994 February

(C) 1994. The American Astronomical Society. All rights reserved. Printed in U.S.A.

\title{
MONTE CARLO SIMULATIONS OF PARTICLE ACCELERATION AT OBLIQUE SHOCKS
}

\author{
MATTHEW G. BARING ${ }^{1}$ \\ Laboratory for High Energy Astrophysics, Code 665, NASA/Goddard Space Flight Center, Greenbelt, MD 20771 \\ DONALD C. ELLISON \\ Department of Physics, North Carolina State University, Box 8202, Raleigh NC 27695 \\ AND \\ FRANK C. JONES \\ Laboratory for High Energy Astrophysics, Code 665, NASA/Goddard Space Flight Center, Greenbelt, MD 20771 \\ Received 1993 March 8; accepted 1993 July 9
}

\begin{abstract}
The Fermi shock acceleration mechanism may be responsible for the production of high-energy cosmic rays in a wide variety of environments. Modeling of this phenomenon has largely focused on plane-parallel shocks, and one of the most promising techniques for its study is the Monte Carlo simulation of particle transport in shocked fluid flows. One of the principal problems in shock acceleration theory is the mechanism and efficiency of injection of particles from the thermal gas into the accelerated population. The Monte Carlo technique is ideally suited to addressing the injection problem directly, and previous applications of it to the quasi-parallel Earth bow shock led to very successful modeling of proton and heavy ion spectra, as well as other observed quantities. Recently this technique has been extended to oblique shock geometries, in which the upstream magnetic field makes a significant angle $\Theta_{B 1}$ to the shock normal. In this paper, spectral results from test particle Monte Carlo simulations of cosmic-ray acceleration at oblique, nonrelativistic shocks are presented. The results show that low Mach number shocks have injection efficiencies that are relatively insensitive to (though not independent of) the shock obliquity, but that there is a dramatic drop in efficiency for shocks of Mach number $\mathbf{3 0}$ or more as the obliquity increases above $15^{\circ}$. Cosmic-ray distributions just upstream of the shock reveal prominent bumps at energies below the thermal peak; these disappear far upstream but might be observable features close to astrophysical shocks.
\end{abstract}

Subject headings: acceleration of particles — cosmic rays — shock waves

\section{INTRODUCTION}

Particle acceleration at shocks is expected to be an important process at many astrophysical sites for three main reasons. First, collisionless shocks are widespread in tenuous plasmas and often contain large amounts of energy; second, at least in quasi-parallel shocks (where the upstream magnetic field is nearly aligned with the shock normal, i.e., $\Theta_{B 1} \sim 0^{\circ}$ ), no special conditions are required for shocks to pick up and accelerate background ions (e.g., Jones \& Ellison 1991); and third, shock acceleration is intrinsically efficient. Evidence for the existence of shocks and accompanying particle acceleration comes from direct spacecraft observations made in the heliosphere at planetary bow shocks, cometary shocks, and interplanetary shocks, and from indirect means such as observations of solar flares and radio and X-ray observations of supernova remnants (SNRs) and extragalactic radio jets.

The first-order Fermi mechanism has been the most popular model of shock acceleration, having modeled, with good accuracy, observations of diffuse ions accelerated at the quasi-parallel Earth bow shock (e.g., Ellison, Möbius, \& Paschmann $1990 \mathrm{~b}$ ), particles and waves observed at interplanetary shocks (e.g., Lee 1983), and the overall energetics of cosmic rays if accelerated in SNRs (e.g., Markiewicz, Drury, \& Völk 1990).

\footnotetext{
${ }^{1}$ NAS-NRC Research Associate.
}

Most theoretical work on the first-order Fermi mechanism has focused on plane-parallel shocks ( see, for example, reviews by Drury 1983; Blandford \& Eichler 1987; Jones \& Ellison 1991). However, most shocks in nature are expected to be oblique, and further, test particle calculations have suggested that oblique shocks may accelerate particles more rapidly than quasi-parallel ones (e.g., Jokipii 1987; Decker 1988; Ostrowski 1988). Such rapid acceleration may have important consequences in many areas of astrophysics including cosmic-ray acceleration in SNRs where severe limits, well below commonly observed cosmic-ray energies, have been placed on the maximum particle energy obtainable, due to the finite age of the blast wave (Lagage \& Cesarsky 1983; Jokipii \& Morfill 1987).

Theoretical determinations of accelerated particle distributions in oblique shock geometries have to date focused on the test particle limit, where the cosmic rays do not modify the shock hydrodynamics. Kirk \& Heavens (1989) and Ballard \& Heavens (1991) obtain numerical solutions to a form of the relativistic cosmic-ray transport equation that takes into account particle anisotropies in the flow. They find that subluminal relativistic shocks generate flatter cosmic-ray distributions than do nonrelativistic ones, largely because of the ion anisotropies in the shock region. An implication of this is that oblique relativistic shocks create flatter distributions than plane-parallel shocks, since increasing the obliquity increases 
the speed of the flow in the shock frame where the flow traces the field lines (the so-called de Hoffmann-Teller frame). Test particle Monte Carlo simulations in plane-parallel (Ellison, Jones, \& Reynolds 1990a) and oblique (Ostrowski 1991) shock geometries confirmed these semianalytic results. However, flat spectra imply more efficient acceleration, and nonlinear Monte Carlo simulations of relativistic parallel shocks (e.g., see Jones \& Ellison 1991), which include injection of cosmic rays and their modification of the shock hydrodynamics, reveal that relativistic shocks produce much steeper spectra than obtained in the test particle calculations.

The oblique shock studies of Kirk \& Heavens (1989) and Ballard \& Heavens ( 1991 ) use the adiabatic approximation to effect particle interactions with the shock discontinuity, a simplification that is adopted in the simulations here. This approximation was not made by Ostrowski (1991), who traced particle gyromotions exactly and demonstrated that the adiabatic approximation made accurate predictions of the spectral index except for mildly relativistic or relativistic shock speeds. All of these authors considered only particles well above thermal energies, specifically those with momentum per unit mass much in excess of the flow speed, which describe the power-law portion of the cosmic-ray spectrum. The Monte Carlo technique here improves upon this limitation by injecting particles directly from the low-energy upstream thermal population, and thereby has the capability of assessing the overall efficiency of Fermi acceleration.

In this paper we elaborate on some of the test particle results from Monte Carlo simulations at oblique shocks that were presented in Baring, Ellison, \& Jones (1993), including the observed rapid decrease of injection efficiency with increase in obliquity for high sonic Mach number shocks. A detailed comparison between upstream and downstream spectra is made. It is found that cosmic-ray distributions just upstream of the shock exhibit prominent features below thermal energies; these features might be observable features close to astrophysical shocks. Subsequent work will include cross-field diffusion in the limit of strong scattering (in progress), the effects of nonadiabaticity and a generalization to relativistic shocks. This will permit extensive comparison with the work of Ostrowski (1991), who includes these developments but has no injection from a thermal population. A major development of our technique will be to then examine the effects of the backreaction of the accelerated particles on the shock structure, investigating how this reaction modifies the injection effciency and the spectral index of the accelerated population.

\section{THE MONTE CARLO METHOD}

The Monte Carlo simulation of particle acceleration at plane-parallel shocks has been described in detail previously (e.g., Ellison, Jones, \& Eichler 1981; Jones \& Ellison 1991), and its extension to oblique shock geometries has been detailed in Baring et al. (1993). The method treats all particles as a single population and follows their time histories in the environs of a shock, allowing them to diffuse, as if scattering off magnetic irregularities anchored in the fluid, with a phenomenological mean free path in the fluid frame of

$$
\lambda_{F}=\lambda_{0}\left(\frac{R}{R_{1}}\right)^{\alpha}\left[\frac{\rho_{1}}{\rho(x)}\right],
$$

where $R=p_{F} c /(Z e)$ is the rigidity of a particle of fluid frame momentum $p_{F}$ and charge $Z e, \rho$ is the plasma density, and $x$ defines distance measured along the shock normal. Rigidities and density are scaled by their values far upstream, i.e., $R_{1}=$ $m_{p} u_{1 x} c / e$ and $\rho_{1}=\rho(x=-\infty)$, where $u_{1 x}=u_{x}(x=-\infty)$ is the upstream flow velocity assumed to lie along the shock normal, and the charge number is set to $Z=1$. Here $\lambda_{0}$ is an input parameter that is taken to be proportional to the gyroradius $r_{g 1}=m_{p} u_{1 x} /\left(e B_{1}\right)$, of an upstream particle. The ratio $r_{g 1} / \lambda_{0}$ is chosen to be much less than unity in this paper so that crossfield diffusion is negligible. Hereafter, the subscript 1 (2) will always indicate upstream (downstream) values.

Comparisons with bow shock observations (Ellison et al. 1990 b) indicate that $1 / 2<\alpha<3 / 2$. Plasma simulations (Giacalone, Burgess, \& Schwartz 1992a) suggest a mean free path obeying equation (1) with $\alpha \sim 2 / 3$. Clearly a variety of microphysics can be incorporated in specifying $\lambda_{F}$ (which defines the particle turnaround length scale), and both pitch angle diffusion (small-angle scattering) and large-angle scattering can be described within the simulation. Therefore the attractiveness of this approach is that the overall nature of the diffusive acceleration process can be approximately modeled without the burden of CPU intensive computations of the underlying physical plasma mechanisms. This simplification is strongly motivated by the success of the Monte Carlo technique in simulating spectra at Earth's bow shock (Ellison et al. 1990b). All results presented here are for $\alpha=1$.

An important property of our model is that no distinction is made between thermal and superthermal ions: all test particles obey equation (1), and as the accelerated particles start off as thermal ones, injection is automatically included in the model. One consequence of this treatment of injection is that at later stages of code development, when the accelerated ions will be allowed to influence the shock hydrodynamics, a self-consistent model of the shock structure and particle acceleration will be achieved. Such internal consistency has been incorporated in Monte Carlo modeling of both nonrelativistic and relativistic plane-parallel shocks (see Jones \& Ellison 1991 and references therein). Particles are allowed to travel between scatterings for a time that is exponentially distributed about the time $t_{c}=\lambda_{F} / v_{F}$, where $v_{F}$ is the particle speed in the fluid frame. In this paper we treat only large-angle scattering: the pitch angle cosine in the fluid frame, $\mu_{F}=\cos \theta_{F}$, where $\theta_{F}$ is the angle between the particle's velocity and the magnetic field, is randomized in each collision, resulting in isotropic scattering.

A major simplification of the Monte Carlo method is to use a guiding-center approximation to the particle transport and at the same time move particles in the de Hoffmann-Teller (HT) frame (de Hoffmann \& Teller 1950), where the drift electric field $\boldsymbol{E}=(\boldsymbol{u} / c) \times \boldsymbol{B}$ is transformed to zero. In the HT frame, the fluid flow is everywhere parallel to the magnetic field, implying a simple description of particle convection. The code calculates only the position of a particle's guiding center, which moves along the field lines, ignoring details of the particle gyromotions except where relevant (Baring et al. 1993). This simplification renders the simulation analogous to the earlier one-dimensional plane-parallel codes (e.g., Jones \& Ellison 1991), and yields a distinct advantage of speed over the Monte Carlo method of Ostrowski ( 1991) and plasma simulation approaches (e.g., Quest 1988), where the exact particle gyrohelices in the magnetic field are calculated. 
The usual hydrodynamic quantities of the downstream fluid are determined from those in the upstream region by the full MHD Rankine-Hugoniot relations (e.g., Decker 1988; Ballard \& Heavens 1991). However, here the magnetic field is chosen to be low enough to be dynamically unimportant (i.e., we treat only shocks of high Alfvénic Mach numbers $\mathscr{M}_{\mathrm{A}}$ ), so that the jump conditions reduce to familiar nonrelativistic forms (e.g., see eqs. (3.4) - (3.6) in Jones \& Ellison 1991). All characteristics of the shocks therefore depend only on the shock compression ratio $r=u_{1 x} / u_{2 x}$, and the sonic Mach number $\mathscr{M}_{s}=$ $\left[m_{p} u_{1 x}^{2} /\left(\gamma k T_{1}\right)\right]^{1 / 2}$, where $T_{1}$ is the upstream temperature and $\gamma=5 / 3$ is the adiabatic index. For the simulations here, these input parameters are chosen to be typical of middle-aged supernova remnants, although they can easily be scaled to suit models of Earth's bow shock or other astrophysical environments. Particles are allowed to escape the simulation once they reach an energy $E_{\max }$ in the normal incidence (NIF) frame (where the upstream plasma velocity is normal to the shock), taken to be $10^{4} \mathrm{keV}$ here.

It is assumed that when particles interact with the shock they conserve their adiabatic invariant $p_{\perp}^{2} / B$. This approximation is a convenient description that assumes that the magnetic field varies smoothly on spatial scales larger than the thermal gyroradius. For $\delta B \ll B$, it is good in the sense that its predictions of various particle properties, such as energy and pitch angle following a particle-shock interaction, correspond quite well to the statistical average obtained from exact numerical simulations that include the details of the particle's gyration (e.g., Hudson 1965; Terasawa 1979). The adiabatic approximation simplifies the treatment of particle transport across the shock significantly. Drury ( 1983 ) showed that this approximation follows from the assumption that particles have random gyrophases when incident upon the shock.

The adiabatic approximation guarantees that our simulations reproduce a key feature of the diffusion approximation, namely that the cosmic-ray spectral index for high-velocity particles is independent of the obliquity of the shock. This important result can be obtained either directly from the diffusion equation (e.g., see Jones \& Ellison 1991), or via an individual particle approach (Drury 1983), which is modeled in these Monte Carlo simulations. The differential cosmic-ray spectrum for particle speeds $v \gg u_{1 x}$ is

$$
f(v) \propto v^{-\sigma}, \quad \sigma=\frac{r+2}{r-1}
$$

where $r=u_{1 x} / u_{2 x}$ is the shock compression ratio. The spectral index in eq. (2) is purely a consequence of the approximate particle isotropy everywhere in both the fluid and shock frames and is also obtained when cross-field diffusion is included (Jones 1994).

Note that for relativistic shocks, the particle speed no longer far exceeds the flow speed and significant particle anisotropies arise, yielding a spectral index $\sigma$ that deviates substantially from the value in equation (2) (Kirk \& Heavens 1989). In the case of test particle plane-parallel relativistic shocks, the Monte Carlo simulations agree closely with Kirk and Heavens's semianalytic determination of the cosmic-ray spectral index (Ellison et al. 1990a). However, as mentioned above, nonlinear models yield substantially different results from the test particle case.

\section{SIMULATION RESULTS}

The principal results of the simulations for the case of negligible cross-field diffusion (see Baring et al. 1993) are presented in Figure 1, which shows omnidirectional energy fluxes, $\tilde{\mathscr{T}}_{0}(E)$, measured in the normal-incidence frame (NIF) downstream from the shock. These fluxes are related to the cosmic-ray distribution function by $\mathscr{F}_{0}(E) d E=4 \pi v f(v) d v$, where $E=m_{p} v^{2} / 2$; they are normalized to unit particle injection upstream. The spectra are calculated at positions more than a few times $\lambda_{0}$ downstream from the shock where isotro-
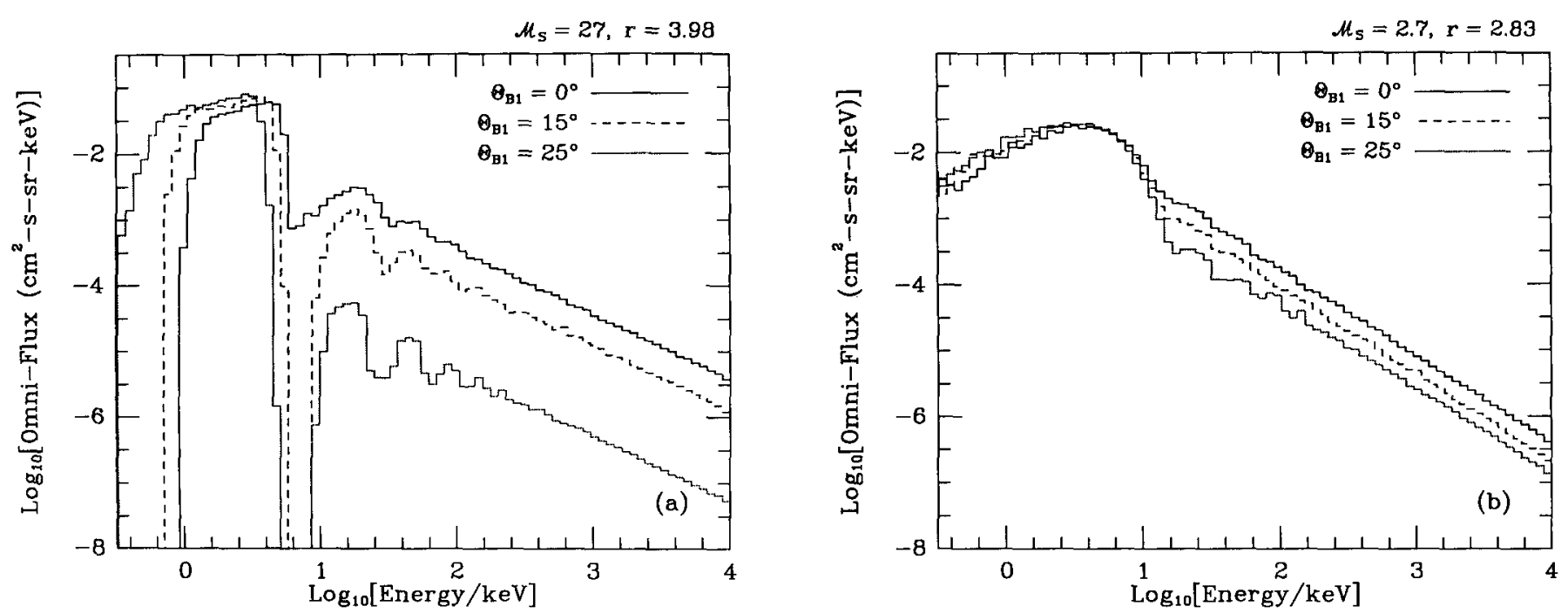

FIG. 1.-Omnidirectional energy fluxes, normalized to unit particle injection upstream and measured downstream of the shock, of $(a)$ high and $(b)$ low Mach number shocks of speed $1000 \mathrm{~km} \mathrm{~s}^{-1}$ in the NIF, for three different shock obliquities (see Baring et al. 1993). Particles were injected into the upstream region, with temperatures $T_{l}=10^{5} \mathrm{~K}\left(\mathscr{M}_{s}=27\right)$ and $T_{1}=10^{7} \mathrm{~K}\left(. \mathscr{M}_{s}=2.7\right)$, and were allowed to escape either far downstream or when their energies exceeded $10^{4} \mathrm{keV}$. A dramatic reduction in injection efficiency with increasing obliquity is evident in the high Mach number case $(a)$ with the normalization dropping by about a factor of 100 between $\Theta_{B 1}=0^{\circ}$ and $25^{\circ}$. In all cases, the power-law indices are within statistical uncertainties of those expected from eq. (3). 
pization of the particles in the fluid frame upon scattering yields spectra that are virtually independent of distance from the shock. Particle splitting was used in the simulations (see Ellison et al. 1990a) to obtain statistics equivalent to approximately 1000 particles at all energies.

As is clear from Figure 1, the Mach number, or equivalently, the preshock temperature, strongly influences the injection efficiency: the decrease in normalization of the high-energy power-law with increasing obliquity is more prominent for higher Mach numbers. At high energies, all spectra show the characteristic power-law distribution that is expected when the acceleration process becomes diffusive. To within statistical errors and independent of obliquity, we obtain the energy spectral index, $\sigma_{E}$, defined by $\mathscr{F}_{0} \propto E^{-\sigma_{E}}$, which for nonrelativistic particles is related to $\sigma$ of equation (12) by $\sigma_{E}=\sigma / 2$ :

$$
\sigma_{E}=-\frac{d \log _{e} \overline{\mathscr{F}}_{0}}{d \log _{e} E}=\frac{1}{2} \frac{r+2}{r-1} .
$$

The high and low Mach number cases have compression ratios of $r=3.98$ and 2.83, and indices of $\sigma_{E}=1.00$ and 1.32, respectively. Values for the relative normalizations of the spectra in Figure 1 are presented in Baring et al. (1993).

In the $\cdot n_{s}=27$ case (Fig. $1 a$ ), the efficiency drops dramatically when $\Theta_{B 1} \gtrsim 15^{\circ}$ and becomes close to zero when $\Theta_{B 1} \gtrsim$ $30^{\circ}$. The reason for this threshold is as follows. For absolutely cold upstream plasmas, particles convect along the field lines into the shock at speed $u_{\mathrm{tHT}}$ in the HT frame, and upon scattering in the downstream region (there is no reflection at the shock ) assume a fluid frame speed of $v_{F}=u_{1 \mathrm{HT}}-u_{2 \mathrm{HT}}$. If this speed is less than the downstream flow speed $u_{2 \mathrm{HT}}$, the particles can never return to the shock and the acceleration process is shut off. Remembering that $u_{1 x}=u_{1 \mathrm{HT}} \cos \Theta_{B 1}, u_{2 x}=$ $u_{2 \mathrm{HT}} \cos \Theta_{B 2}$ and that the compression ratio is given by $r=$ $u_{1 x} / u_{2 x}$, the condition for this termination of injection of particles from the upstream gas into the diffusive acceleration pro- cess is

$$
\frac{\cos \Theta_{B 1}}{\cos \Theta_{B 2}} \geq \frac{r}{2} \approx 2
$$

for strong shocks $(r \approx 4)$ of infinite $\mathscr{M}_{s}$; injection then truly ceases for obliquities $\Theta_{B 1} \gtrsim 30^{\circ}$. The $\mathscr{U}_{s}=27$ case in Figure $1 a$ closely approximates this idealized case, though there is always some (albeit small) injection for obliquities above $30^{\circ}$. For the $\mathscr{H}_{s}=2.7$ examples in Figure $1 b$, this critical angle is around $55^{\circ}$, above which production of the power law is virtually (but not totally) suppressed. This termination of particle injection will not occur when cross-field diffusion is significant.

Note that the injection efficiency becomes less sensitive to changes in the shock obliquity at low Mach numbers (higher temperatures) because the probability of return to the shock from the downstream region is enhanced, and also many more particles have pitch angles which allow them to be reflected in their first encounter with the shock. The introduction of crossfield diffusion is expected to diminish this sensitivity.

Other features of the spectra merit mention. The principal downstream peak is due to shock-heated particles that have crossed the shock just once, while the secondary peak at higher energies is a second "harmonic" formed by particles that have crossed the shock only twice in the downstream direction. Higher order peaks merge into a continuum as the acceleration process becomes diffusive. Also apparent in Figure $1 a$ are gaps between the first and second spectral peaks for the oblique cases resulting from particle reflection. For high $\mathscr{M}_{s}$, particles that have scattered upstream after crossing the shock once in each direction have high fluid frame speeds $\left(v_{F} \simeq u_{1 \mathrm{HT}}\right)$, so that reflection is very likely when the shock is oblique. Those particles that are then transmitted downstream generally have higher NIF speeds, while the reflected ones receive an extra velocity kick in the upstream region before convecting downstream. The net effect is that the lower energy portion of the
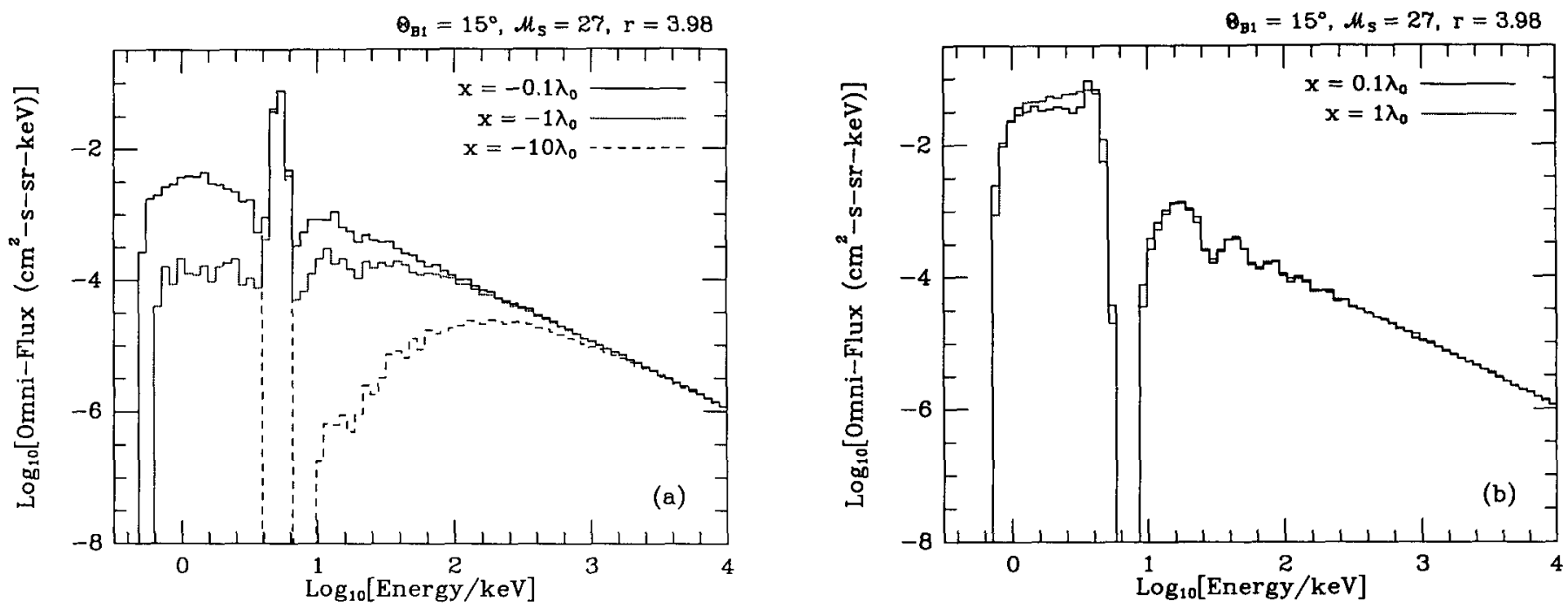

FIG. 2.-Omnidirectional energy fluxes in the NIF, for the low-temperature $\left(T_{1}=10^{5} \mathrm{~K}\right), \Theta_{B 1}=15^{\circ}$ case in Fig. 1 measured at various positions ( $a$ ) upstream $(x<0)$ and $(b)$ downstream $(x>0)$ of the shock. The position $x$ is expressed in terms of the "thermal" mean free path $\lambda_{0}$ defined in eq. (1). The prominent peak in the upstream spectra is just the cold upstream gas, while the broad spectrum around it is generated by particles that have returned from the downstream region and so is less prominent further upstream. Very little change occurs in the downstream spectrum for $x \gtrsim \lambda_{0}$. 
second peak is suppressed so that the peak is narrower and a gap between it and the principal peak appears. This phenomenon is manifested in higher order peaks as well (see Fig. 1a), where these peaks are broad when $\Theta_{B 1}=0^{\circ}$ (and therefore merge more easily into a continuum) but are comparatively narrow and resolved for $\Theta_{B 1}=25^{\circ}$. The principal and secondary peaks are smeared out by thermal broadening when $\mathscr{M}_{s}=$ 2.7 , and the gap between them (for $\Theta_{B 1}>0^{\circ}$ ) disappears.

A comparison of cosmic-ray distributions at different distances from the shock, both upstream and downstream, is made in Figure 2. The upstream spectra in Figure $2 a$ show a prominent narrow thermal peak, which is the cool gas injected far upstream, together with a broad continuum of particles that have returned (at least once) from the downstream region. This continuum diminishes rapidly at positions further upstream since particles encounter great difficulty in convecting against the flow. At high energies, the same power law as in the downstream distribution of Figure $2 b$ is generated, since energetic particles have mean free paths much larger than $\lambda_{0}$. The population at energies lower than the thermal peak is comprised of slow particles that have been downstream generally only once: they form a prominent and somewhat narrow peak for plane-parallel shocks and should be an observable feature of acceleration at shocks. Such a slow population also appears as part of the broad quasi-thermal peak downstream (Fig. 2b); when $x=0.1 \lambda_{0}$, this peak shows substructure that is suggestive of the upstream thermal peak. In fact as $|x|$ becomes much less than $\lambda_{0}$, continuity guarantees that the upstream and downstream spectra approach each other. Note also that the downstream distribution varies little when $x \gtrsim \lambda_{0}$.

Finally, Figure 3 displays passage of a typical particle through an oblique shock region during a simulation run where particle splitting was suppressed in order to secure the identity of the particle. The particle's motion relative to the shock front bears considerable similarity to that obtained in hybrid plasma simulations (Giacalone et al. 1992b). Before the first shock encounter in Figure 3, the particle convects slowly into the shock since $v_{F} \lesssim u_{1 x}$. After a few shock interactions it quickly gains energy, and when $v_{F} \gg u_{1 x}$, it is observed that the particle can spend extended periods of time either upstream or downstream without a single shock crossing. Notice also that at these higher energies, the back-and-forth motion of the particle is more exaggerated upstream of the shock, and the particle tends to convect further from the shock in the upstream region than downstream: this reflects the greater flow density and field obliquity in the downstream region. Also, the velocity increments at shock encounters in Figure 3, which are always positive, appear to be largely uncorrelated with the value of $v_{F} / u_{1 x}$ (this can be demonstrated analytically). Reflections play a prominent role, occurring for six out of the 14 encounters of the shock from the upstream side in Figure 3.

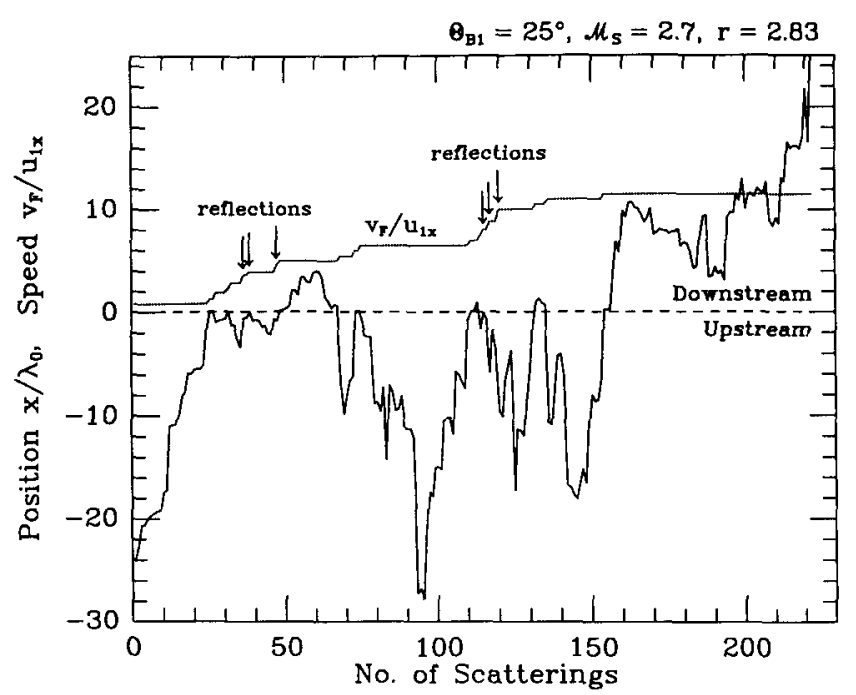

FIG. 3.-The passage of a typical particle during a simulation run for $\Theta_{B 1}=25^{\circ}$ is depicted, with the solid line representing the position $x$ of the particle at each scattering (the $x$-axis effectively represents time). Again $x$ is expressed in terms of the "thermal" mean free path $\lambda_{0}$ defined in eq. (1). Here $x<0$ defines the upstream region. The dotted line shows the particle speed $v_{F}$ in the local fluid frame in units of $u_{1 x}$; its monotonic behavior is the characteristic feature of the diffusive acceleration mechanism. The energy gains that are due to reflections are marked accordingly.

\section{SUMMARY}

In conclusion, here we have presented the main features of our Monte Carlo simulation of test particle acceleration at oblique shocks in the limit of no cross-field diffusion. Particles are automatically injected into the diffusive acceleration mechanism in crossing the shock, and the simulation can make predictions of the efficiency of this injection (Baring et al. 1993), which decreases rapidly with an increase in obliquity for high Mach number shocks, effectively shutting off acceleration when $\Theta_{B 1} \gtrsim 30^{\circ}$ for cold upstream gases. The spectra depicted have high-energy power-law spectral indices that are independent of obliquity. Cosmic-ray distributions just upstream of the shock reveal prominent bumps at energies below the thermal peak; these should be observable features. Baring et al. (1993) observe that the simulation agrees with Jokipii's (1987) prediction of shorter acceleration times in oblique shocks. The determination of injection efficiencies and acceleration times will be strongly modified by the inclusion of crossfield diffusion, and work is in progress to address this important parameter regime.

We thank Stephen Reynolds for many helpful discussions and suggestions relating to this work. Partial support for this work came from NASA grants NAG 5-1042, NAG 5-2254, NAGW 2001, and NSF grant AST-88-17567.
Ballard, K. R., \& Heavens, A. F. 1991, MNRAS 251, 438 Baring, M. G., Ellison, D. C., \& Jones, F. C. 1993, ApJ 409, 327 Blandford, R. D., \& Eichler, D. 1987, Phys. Rep., 154, 1 Decker, R. B. 1988, Space Sci. Rev., 48, 195 de Hoffmann, F., \& Teller, E. 1950, Phys. Rev., 80, 692 Drury, L. O’C. 1983, Rep. Prog. Phys., 46, 973

\section{REFERENCES}

Ellison, D. C., Jones, F. C., \& Eichler, D. 1981, J. Geophys., 50, 110 Ellison, D. C., Jones, F. C., \& Reynolds, S. P. 1990a, ApJ, 360, 702 Ellison, D. C., Möbius, E., \& Paschmann, G. 1990b, ApJ, 352, 376 Giacalone, J., Burgess, D., \& Schwartz, S. J. 1992a, in Proc. 26th ESLAB Symposium, Study of the Solar-Terrestrial System (Noordwijk: ESA), 65 
Giacalone, J., Burgess, D., Schwartz, S. J., \& Ellison, D. C. 1992b, Geophys. Res. Lett., 19, 433

Hudson, P. D. 1965, MNRAS, 131, 23

Jokipii, J. R. 1987, ApJ, 313, 842

Jokipii, J. R., \& Morfill, G. 1987, ApJ, 312, 170

Jones, F. C. 1994, ApJS, 90, 61

Jones, F. C., \& Ellison, D. C. 1991, Space Sci. Rev., 58, 259

Kirk, J. G., \& Heavens, A. F. 1989, MNRAS, 239, 995
Lagage, P. O., \& Cesarsky, C. J. 1983, A\&A, 125, 249

Lee, M. A. 1983, J. Geophys. Res., 88, 6109

Markiewicz, W. J., Drury, L. O'C., \& Völk, H. J. 1990, A\&A, 236, 487

Ostrowski, M. 1988, A\&A, 206, 169

-. 1991, MNRAS, 249, 551

Quest, K. B. 1988, J. Geophys. Res., 93, 9649

Terasawa, T. 1979, Planet. Space Sci., 27, 193 